\title{
Contribution of Brain MRI after Head CT in the Evaluation of Acute, Hospitalized Traumatic Brain Injury Patients: An Under-Utilized Resource?
}

\author{
Wassim Malak ${ }^{1 *}$, Muhammad Mustafa Qureshi ${ }^{1}$, Ronald J Killiany ${ }^{1}$, Tejal S Brahmbhatt ${ }^{2}$, \\ Courtney Takahashi ${ }^{3}$ and Asim Mian ${ }^{4}$ \\ ${ }^{1}$ Department of Radiology, Boston University School of Medicine, USA \\ ${ }^{2}$ Department of Surgery, Boston University School of Medicine, USA \\ ${ }^{3}$ Department of Neurology, Subdivision of Neurocritical Care, Boston University School of Medicine, USA \\ ${ }^{4}$ Department of Radiology, Subdivision of Neuroradiology, Boston University School of Medicine, USA \\ *Corresponding author: Wassim Malak, Department of Radiology, Boston University School of Medicine, USA
}

\section{ARTICLE INFO}

Received: March 13, 2020

Published: March 31, 2020

Citation: Wassim M, Muhammad MQ, Ronald JK, Tejal SB, Courtney T, et al. Contribution of Brain MRI after Head CT in the Evaluation of Acute, Hospitalized Traumatic Brain Injury Patients: An UnderUtilized Resource?. Biomed J Sci \& Tech Res 26(5)-2020. BJSTR. MS.ID.004408.

Keywords: Magnetic Resonance Imaging; Computed Tomography; Altered Mental Status; Traumatic Brain Injury; Diffuse Axonal Injury
ABSTRACT

Purpose: Non Contrast Head CT (NHCT) has long been the study of choice for initial imaging of patients presenting with acute Traumatic Brain Injury (TBI). However, MRI has been used more frequently as imaging times have decreased. The purpose of this study was to assess the efficacy of lesion detection between NHCT and MRI as well as to estimate the frequency of MRI utilization in routine clinical practice during the care of acute TBI patients.

Methods: A retrospective cohort of patients presenting with acute TBI to a level 1 trauma center between November 2007 and December 2013 who received an NHCT and subsequently received brain MRI. The electronic medical record (EMR) was reviewed to assess indications for imaging and physical exam findings. NHCT and MRI findings were compared.

Results: There were 318 patients who presented with moderate or severe TBI. Of those, $134 / 318(42.1 \%)$ had findings visualized on bMRI not detected on NHCT, most common being diffuse axonal injury (DAI) $(59 / 134 ; 44.0 \%)$ and infarct $(37 / 134$; $27.6 \%)$. The bMRI was most often ordered for an abnormal clinical exam finding (73\%). The most common abnormality was altered mental status (44.8\%). Among all patients admitted to the inpatient level of care for acute TBI, only $16 \%$ receive an MRI brain during their admission.

Conclusion: In summary, our findings show that brain MRI is able to detect more lesions than NHCT in acute TBI patients. Our data show that brain MRI may be an underutilized resource in these patients.

Abbreviations: AMS: Altered Mental Status; bMRI: Brain Magnetic Resonance Imaging; CT : Computed Tomography; DAI: Diffuse Axonal Injury; DE: Driven Equilibrium; DWI: Diffusion-Weighted Image; ED: Emergency Department; EMR: Electronic Medical Record; FFE: Fast Field Echo; FLAIR: Fluid Attenuated Inversion Recovery; FOV: Field of View; GCS: Glasgow Coma Scale; MPRAGE: Magnetization Prepared Rapid Gradient Echo; MRI: Magnetic Resonance Imaging; NHCT: Non-Contrast Head Computed Tomography; PACS: Picture Archiving and Communication System; RIS: Radiology Information System; SE: Spin Echo; STIR: Short Tau Inversion Recovery; TBI: Traumatic Brain Injury; TE: Time to Echo; TR: Repetition Time 


\section{Introduction}

Imaging plays a crucial role in the initial evaluation of moderate and severe Traumatic Brain Injury (TBI) patients. NonContrast Head CT (NHCT) has long been the initial step of imaging management in the acute traumatic brain injury patient [1] because it has high sensitivity and specificity for intracranial bleeds, cerebral edema, and skull fractures and it is quick and cost-effective. Despite its utility, head CT has limitations: it may underestimate the degree of injury in the acute setting, and very small amounts of blood may be missed due to volume averaging or artifact that can obscure hemorrhages [2,3]. Previous studies show the efficacy of MRI in patients who present with TBI. Prior retrospective data from emergency department patients presenting with TBI demonstrate $96.4 \%$ sensitivity and $63.4 \%$ sensitivity with MRI and CT, respectively [4,5]. MRI was more sensitive for detecting contusion, shearing injury, subdural hematoma, epidural hematoma, and sinus involvement. CT was more sensitive for detecting skull fracture [5]. Our hypothesis is twofold: first, we hypothesize that because of NHCT's limitations, brain MRI (bMRI) may be the superior study for acute TBI management. Second, we hypothesize that brain MRI is underutilized in clinical practice, given its sensitivity and specificity for lesion detection. Brain MRI's sensitivity may result in earlier detection of acute findings.

\section{Methods and Materials}

\section{Subjects}

The Boston University IRB approved this study prior to any data collection. This retrospective study identified 318 sequential patients between November 2007 and December 2013. Inclusion criteria are as follows: Adult patients $>18$ years of age (without an upper age limit), male and female patients included, head CT ordered with a trauma indication, hospital admission for further management. Exclusion criteria are as follows: MR imaging performed prior to head CT, incomplete MR imaging, and discharge from the ED or observational level admission only. In order to determine the frequency of MRI utilization in routine clinical practice, a convenience sample of sequentially admitted patients from the Boston Medical Center Trauma Database was included. The trauma database identified 1235 patients between January 2016 and January 2018. The inclusion criteria for the second query were as follows: adult patients admitted for at least 24 hours to the inpatient setting for traumatic brain injury. Six hundred ten subjects met inclusion criteria.

\section{Procedures}

Radiological Information System (RIS) produced a report that identified all patients who received a trauma protocol NHCT in conjunction with brain MRI between November 2007 and December 2013. The brain MRI could be performed any time after the NHCT and before the patient was discharged from the hospital. If multiple NHCTs were ordered, we compared the NHCT that was performed immediately prior to the bMRI. We chose to compare findings from the most recent NHCT so that delayed findings (e.g. delayed or blossoming contusions) would be accurately identified on both imaging studies. In order to determine the frequency of MRI ordering in a random two-year period, we ran a query through the Boston Medical Center Trauma Database to determine the number of patients admitted for traumatic injuries. Initially, 1235 subjects met criteria for admission, and the search was refined to include subjects who had TBI included as one of their injuries. TBI was defined by the treating clinician - if present, the clinician would subsequently fill out database fields documenting Glasgow Coma Scale (GCS). If absent, these fields are blank. Six hundred ten subjects met the criteria for TBI. The subjects with TBI were reviewed with the Philips Search Tool (query: CT, MRI) to determine which subjects received head CTs and bMRI.

\section{Data Acquisition}

The initial NHCT was performed on a GE Lightspeed VCT 64-slice CT scanner, $250 \mathrm{~mA}, 120 \mathrm{kV}$ with $5.0 \mathrm{~mm}$ slice thickness with 1.25 and $2.5 \mathrm{~mm}$ axial and coronal reconstructions in both soft tissue and bone algorithms, which scans from the hard palate to the cerebral vertex in a gantry angle parallel to the hard palate. The subsequent bMRI was performed on either of our two MRI scanners, Philips Achieve 1.5T and Philips Ingenia 3.0T. The noncontrast brain protocol includes a axial DWI $(b=0,1000$; TR/ $\mathrm{TE}=4187 / 68.8 \mathrm{msec}$, slice thickness $=5 \mathrm{~mm}$, matrix $=256 \times 256 \mathrm{~mm}$, FOV=240), axial FLAIR (6000/100msec; 5mm; 352/352mm; 220), axial STIR (6135.9/90msec; 5mm; 336x336mm; 220), axial FFE/ GRE (1395.6/23msec; 5mm; 320x320; 220), sagittal 3D MPRAGE, a high definition T1 sequence $(8.5 / 4.2 \mathrm{msec} ; 1.8 \mathrm{~mm}$; 336x336mm; 240), axial dual echo proton density/T2 (2013.3/9.5 [PD] or 90 [T2]msec; 448x448 mm; 220), axial SE T1 (800/10msec; 5mm; $448 \times 448 \mathrm{~mm} ; 220$ ).

\section{Terms and Measures}

We reviewed clinical notes to determine the patient presentation and brain MRI indications. Patients were categorized as moderate or severe TBI. Moderate TBI was defined as GCS9-12 and an NHCT without changes due to the mechanism of injury. Severe TBI was defined as an abnormal NHCT (i.e., any finding that was deemed directly related to the mechanism of injury) and/or GCS 3-8. Reports and progress notes in PACS and the EMR were reviewed to determine the indication for each bMRI and to assess the significant findings detected on bMRI not initially detected by NHCT on admission. We categorized indications for MRIs as follows: based on clinical findings only, based on admission head CT findings, and indication based on both clinical and radiographic findings on admission head CT (Figure 1). The clinical indications were subsequently subcategorized into Altered Mental Status (AMS), seizure or seizure-like activity, focal deficit, and headache. New MRI findings were defined as any finding not present on the initial head CT. We further went on to categorize findings on MRI 
as follows: clinically relevant or directly related to the trauma regardless of clinical relevance. New findings included diffuse axonal injury, ischemia/infarct, mass lesion, and others (such as hemorrhage or contusion).

\section{Statistical Analysis}

To compare the incidence of findings from NHCT to overall findings from bMRI, a one-sample test of proportion was conducted to test the hypothesis that the observed incidence of findings from bMRI was different from the incidence observed with NHCT. Exact 95\% confidence interval (95\% CI) was calculated for the bMRI proportion. A P-value of less than 0.05 was considered statistically significant. All statistical analyses were performed using SAS 9.4 (SAS Institute, Cary, NC).

\section{Results}

The most common clinical indications for obtaining a bMRI were AMS (44.8\%), focal neurologic deficit (36.5\%), and seizure or seizure-like activity (19.1\%), (Figure 1). AMS had the highest percentage of positive studies (61.9\%). The average number of days between the most recent NHCT and bMRI was 4.7 days (Median: 2, Min: 0, Max: 88). In the convenience sample, 610 patients had TBI as one of their injuries. $510 / 610(83.6 \%)$ received NHCT. 100/610 $(16.4 \%)$ received bMRI. $82 / 610(13.4 \%)$ received bMRI as a followup study after a head CT.

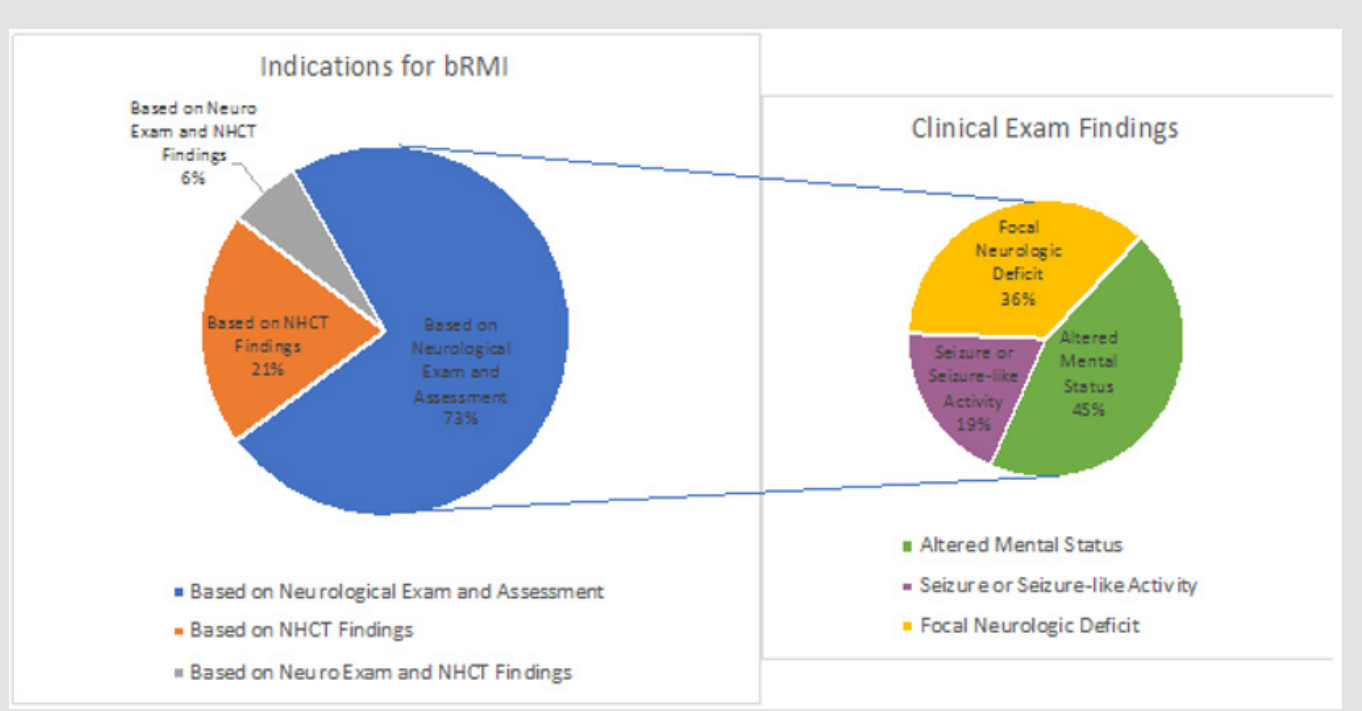

Figure 1: Left chart: Summary of indications of bMRI after NHCT performed in patients presenting with acute TBI. Right chart: Further subdivides the types of findings on neurological exam and assessment mentioned in the chart on the left.

\section{All Patients (Moderate and Severe TBI)}

A total of 318 brain MRIs for TBI were obtained over a 6-year period. In patients, regardless of TBI severity, 178/318 (56.0\%) had findings visualized on NHCT. A total of 214/318 (67.3\%) findings were visualized on bMRI of which 134/318 (42.1\%) had additional findings visualized on bMRI not captured on NHCT (Figures $2 \& 3$ ).
The overall findings observed from bMRI were significantly higher (67.3\%: 95\% CI 61.8\% to $72.4 \%$ ) than observed on an NHCT, $\mathrm{P}<0.0001$. The most common findings detected by bMRI were diffuse axonal injury (DAI) (59/134; 44.0\%) and infarct (37/134; $27.6 \%)$. Other findings were grouped together and categorized as mass/lesion/other, which includes hemorrhage, contusions, and incidental masses (Figure 4).

\begin{tabular}{|c|c|c|c|}
\hline Moderate + Severe TBI & bMRI(+) & bMRI(-) & Total \\
\hline CT( $(+)$ & CT(+) bMRI(+) & CT(+) bMRI(-) & Total CT(+) \\
& 98 & 80 & 178 \\
\hline CT(-) & CT(-) bMRI(+) & CT(-) bMRI(-) & Total CT(-) \\
& 36 & 104 & 140 \\
\hline Total & Total bMRI(+) & Total bMRI(-) & \\
& 134 & 184 & \\
\hline
\end{tabular}

Figure 2: Cumulative findings of $\mathrm{CT}$ and bMRI regardless of TBI severity. 


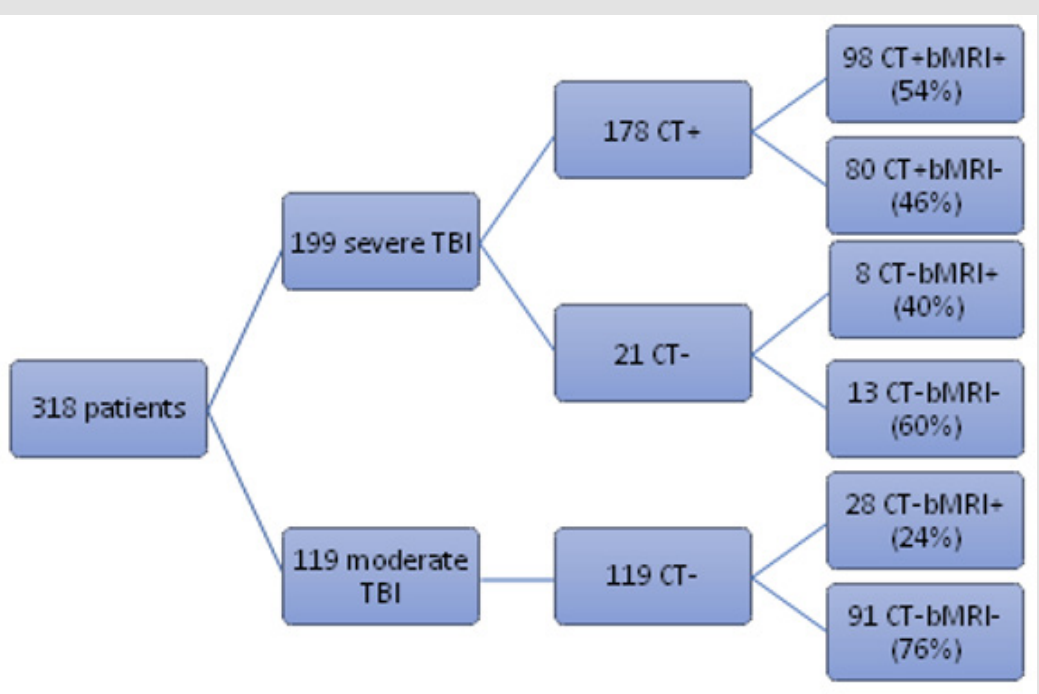

Figure 3: CT and bMRI findings based on TBI severity.

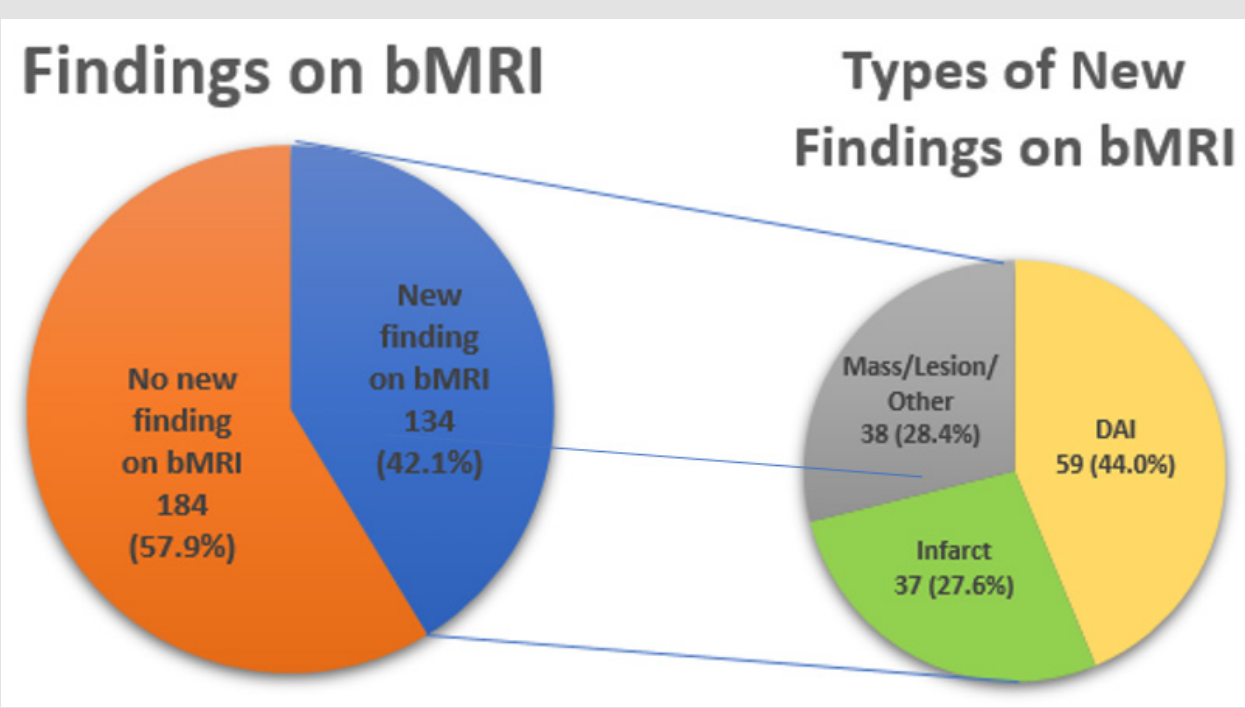

Figure 4: Cumulative bMRI findings for both moderate and severe TBI patients.

\section{Severe TBI Patients}

There were 199 patients categorized as severe TBI (Figure 3). Of these, 178/199 (89.4\%) had findings visualized on NHCT. A total of 186/199 (93.5\%) findings were visualized on bMRI of which 106/199 (53.3\%) had additional findings visualized on bMRI (bMRI+) not captured on NHCT. The overall findings observed from bMRI were not significantly higher (93.5\%: 95\% CI 89.1\% to $96.5 \%$ ) than observed on an NHCT, P=0.060. Twenty-one severe TBI patients had normal NHCT on admission. In this sub-population of negative NHCT severe TBI, 8/21 (38.1\%) had new findings evident only with brain MRI evaluation. Among the 178 severe TBI patients with a positive NHCT, 98/178 (55.1\%) had new bMRI findings.

\section{Moderate TBI Patients}

All 119 moderate TBI patients received NHCT and subsequent brain MRI. In the moderate TBI patients, 28/119 (23.5\%) had findings evident only on brain MRI (Figure 3).

\section{Discussion}

In our study, we observed that patients with moderate and severe TBI often have imaging findings not visualized on the initial head CT. New findings on bMRI were more common in patients with severe TBI, but were still frequent in both groups $53.3 \%$ in severe TBI and $23.5 \%$ in moderate TBI). The most common finding is DAI (44.0\%), the second most common finding is infarct (27.6\%). The bMRI visualized findings on $42.1 \%$ of all patients with TBI regardless of severity that was not seen on NHCT, and the overall findings on bMRI were significantly higher than on NHCT. These results support our hypothesis that brain MRI will reveal additional findings compared to admission head CT. Our study also shows that bMRI is likely underutilized for clinical care. Based on our convenience sampling data, in a two year period, we perform bMRI on $13.4 \%$ of all patients with disease severe enough to warrant inpatient admission. If our study findings are accurate; however, 
then we would expect $42.1 \%$ of all bMRIs to have additional findings. This translates into findings on 257 (42.1\% of 610) patients in a two year time period. It also means that there may be as many as 157 [210 - (82+18)] patients with undiagnosed findings because they did not receive bMRI. While these calculations are speculative, they illustrate the point that there is likely a large gap of patients with missed findings that could have been captured with bMRI. Other studies support this estimate, quoting rates of additional findings on bMRI as high as $67 \%$.

Early identification of DAI is important for patient management because it can influence family counseling and recommendations regarding prognosis. A prospective study performed on patients with confirmed DAI evaluated 6-month outcomes. The study associated DAI with increased risk for both mortality and dependence [6]. These associations can have a significant impact on patients and their families. Most trauma patients are young men in the workforce; the median age in one of the largest trials to date was 39 years [7]. Hence, even small cognitive changes may affect their capability for full-time employment. Because brain MRI findings such as DAI may guide prognosis, clinicians may start post-TBI education earlier with families. Early education allows families more time to plan for rehabilitation stays and possible loss of income [8]. Timely identification and treatment of stroke are paramount for best patient care. Stroke in the setting of trauma may suggest dissection or occult infection [9]. It may also suggest an underlying etiology preceding the traumatic event, such as a stroke leading to a car accident. Most patients who suffer from TBI associated ischemic stroke are polytrauma patients with fractures and abdominal-thoracic injuries. The decision making algorithms for these patients is often complex and requires specialist expertise [10]. Therefore, rapid stroke identification, early specialist consultation, and subsequent stroke treatment will result in better overall patient care.

Our findings are consistent with prior studies. Data from pediatric populations that presented with TBI also show that MRI has a superior ability to pick up radiographic findings missed on head CT [11]. Retrospective data from adult ED patients presenting with TBI showed higher sensitivity of MRI in identifying nonhemorrhagic lesions (DAI, contusion, brainstem injury) but also noted the advantage of CT in patients who are unstable that might need surgery $[12,13]$. There are several limitations to our study. First, this is a single institution, retrospective study limiting the generalizability of our findings. A more extensive study, utilizing data collected from several different institutions would help rectify this limitation in the future. We did not collect any information about gender, past medical history, and pre-existing conditions. It is possible that our sample may have contained a disproportionate number of patients with conditions such as cancer or atherosclerotic disease, thus increasing the likelihood that they would suffer from subsequent intracranial pathology associated with their TBI.
We did not adjust our data to account for the lag time between admission head CT and bMRI acquisition. While less likely, prolonged lag times increase the likelihood that patients may suffer other events unrelated to the original trauma (e.g., second fall in the hospital, arrhythmias). In these rare instances, the brain MRI may be identifying findings outside of the trauma on admission, thus affecting our study results. A future multi-center, observational study with structured pre-specified data elements and imaging collection times would address many of our studies' limitations.

\section{Conclusion}

Patients presenting with acute trauma, more specifically presenting with severe TBI, are more likely to have additional findings on bMRI not visualized on the initial NHCT. Thus earlier imaging with MRI may be beneficial for clinical management, and imaging can be considered within the first week of admission [14]. Our results suggest that bMRI may be an underutilized resource in the acute care of moderate and severe TBI patients.

\section{Conflicts of Interest}

None of the authors have any relevant conflicts of interest to disclose.

\section{References}

1. Rosenfeld JV, Maas AI, Bragge P, Morganti Kossmann MC, Manley GT (2012) Early management of severe traumatic brain injury. Lancet (London, England) 380(9847): 1088-1098.

2. Lee B, Newberg A (2015) Neuroimaging in Traumatic Brain Imaging. NeuroRx J Am Soc Exp Neurother 2(2): 372-383.

3. Barrett JF, Keat N (2004) Artifacts in CT: Recog- nition and Avoidance 1 LEARNING OBJECTIVES FOR TEST 5 CME FEATURE 2004. 24(6).

4. Orrison WW, Gentry LR, Stimac GK, Tarrel RM, Espinosa MC (1994) Blinded Comparison of Cranial CT and MR in Closed Head Injury Evaluation. Community Med 15(2): 351-356.

5. Roguski M, Morel B, Sweeney M, Talan J, Rideout L, et al. (2015) Magnetic resonance imaging as an alternative to computed tomography in select patients with traumatic brain injury: A retrospective comparison. J Neurosurg Pediatr 15 (5): 529-534.

6. Vieira A, Kobeissy FH, Rafaels KA, Sarhane KA, De Cássia R, et al. (2016) Diffuse axonal injury: epidemiology, Outcome and associated risk Factors. Front Neurol 7: 1-12

7. B Farrell, G Lomas, R Mashru, N Ritchie, IRobertsv (2002) The MRC CRASH Trial: study design, baseline data, and outcome in 1000 randomised patients in the pilot phase. n.d.19 (6): 510-514.

8. Gagnon A, Lin J, Stergiou Kita M (2016) Family members facilitating community re-integration and return to productivity following traumatic brain injury - motivations, roles and challenges. Disabil Rehabil 38(5): 433-441.

9. Kowalski RG, Haarbauer Krupa JK, Bell JM, Corrigan JD, Hammond FM, et al. (2017) Acute Ischemic Stroke after Moderate to Severe Traumatic Brain Injury. Stroke 48(7): 1802-1809.

10. Foreman PM, Harrigan MR (2017) Blunt Traumatic Extracranial Cerebrovascular Injury and Ischemic Stroke. Cerebrovasc Dis Extra 7(1): 72-83.

11. Buttram SDW, Garcia Filion P, Miller J, Youssfi M, Brown SD, et al. (2015) Computed tomography vs magnetic resonance imaging for identifying 
acute lesions in pediatric traumatic brain injury. Hosp Pediatr 5(2): 79 84.

12. Lindell L, Gentry R, Godersk JC, Thompson B, Dunn V, et al. (1988) Prospective Comparative Study of Intermediate-Field MR and CT in the Evaluation of Closed Head Trauma. AJR Am J Roentgenol 150(3):673 682.

ISSN: 2574-1241

DOI: $10.26717 / B J S T R .2020 .26 .004408$

Wassim Malak. Biomed J Sci \& Tech Res

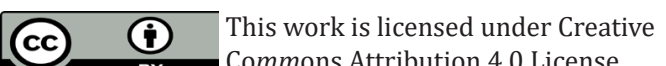

Submission Link: https://biomedres.us/submit-manuscript.php
13. Mutch CA, Talbott JF, Gean A (2016) Imaging Evaluation of Acute Traumatic Brain Injury. Neurosurg Clin N Am 2016. 27 (4): 409-439.

14. Skandsen T, Kvistad KA, Solheim O, Lydersen S, Strand IH (2011) Prognostic value of magnetic resonance imaging in moderate and severe head injury: A prospective study of early MRI findings and one-year outcome. J Neurotrauma 28 (5): 691-699.

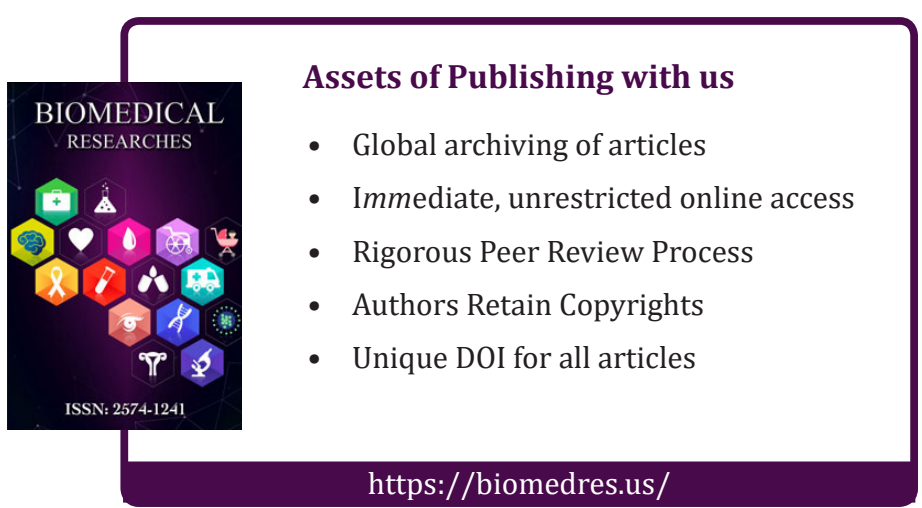

